\section{The Quality of Life Measurement in Recurrent Acute Pancreatitis Needs to be More Accurate}

\author{
Lu Hao, MD ${ }^{1,2}$, Zhao-Shen Li, MD ${ }^{1,2}$ and \\ Liang-Hao Hu, MD ${ }^{1,2}$
}

Am J Gastroenterol https://doi.org/10.1038/s41395 018-0244-z

To the Editor: We read the recently published study on the physical and mental quality of life (QOL) in recurrent acute pancreatitis (RAP) patients [1]. It is a large sample study, which compared the QOL between RAP, chronic pancreatitis (CP), and non-disease controls. However, some of the study is confusing.

In the present study, RAP was defined by evidence of two or more documented attacks of acute pancreatitis (AP), but without imaging or histological evidence of chronic pancreatitis. However, the definition of RAP is still controversial. In some studies, RAP was defined as two or more well-documented separate episodes of pancreatitis that resolved with more than 3 months between attacks [2]. As pancreatic parenchymal changes may persist after the complete normalization of pancreatic enzymes and resolution of patient's symptoms, the definition is less specific if the interval between the acute attacks is short [3]. In some other studies, RAP was defined as more than two acute attacks within 1 year, as acute attacks after a long interval may have different etiologies [4]. Thus, patients included as "RAP" in the present study are "patients with two or more AP attacks" indeed, which are heterogeneous.

Endocrine insufficiency was concluded to have significant effect on Physical
Component Summary in RAP patients. However, RAP patients may have transient endocrine insufficiency during the acute attack, but between episodes of pancreatitis, the blood glucose level was normal [3]. Here comes the question, what point in time the endocrine function was assessed. Is this endocrine insufficiency during acute attack or between episodes of pancreatitis?

The definition of endocrine insufficiency was also unclear. Are patients with impaired glucose tolerance also defined as endocrine insufficiency or just patients with diabetes mellitus (DM) included? Also, there are three types of DM, among which only type $3 c$ is pancreatogenous. DM is a common disease with the prevalence of $0.84 \%$ in the United States, and type $2 \mathrm{DM}$ accounted for $43 \%$ of total diabetes cases [5]. It is difficult to differentiate type $3 \mathrm{c}$ and type $2 \mathrm{DM}$. Thus, it is really confusing how endocrine insufficiency could be identified in the present study.

In conclusion, this is a large sample study about the physical and mental QOL in RAP patients. However, definition of RAP, endocrine insufficiency, and point of time to estimate endocrine function were unclear.

\section{CONFLICT OF INTEREST}

Guarantor of the article: Liang-Hao $\mathrm{Hu}$, MD and Zhao-Shen Li, MD

Specific author contributions: $\mathrm{LH}$ participated in the manuscript drafting. Z-SL and L-HH contributed to the planning and conducting of the study. L-HH and Z-SL contributed equally to this manuscript.

Financial support: None.

Potential competing interests: None.

\section{REFERENCES}

1. Cote GA, Yadav D, Abberbock JA, et al. Recurrent acute pancreatitis significantly reduces quality of life even in the absence of overt chronic pancreatitis. Am J Gastroenterol. 2018;113:906-12.

2. Takuma K, Kamisawa T, Hara S, et al. Etiology of recurrent acute pancreatitis, with special emphasis on pancreaticobiliary malformation. Adv Med Sci. 2012;57:244-50.
3. Romagnuolo J, Guda N, Freeman M, et al Preferred designs, outcomes, and analysis strategies for treatment trials in idiopathic recurrent acute pancreatitis. Gastrointest Endosc. 2008;68:966-74.

4. Khurana V, Ganguly I. Recurrent acute pancreatitis. JOP. 2014;15:413-26.

5. Demmer RT, Zuk AM, Rosenbaum M, et al. Prevalence of diagnosed and undiagnosed type 2 diabetes mellitus among US adolescents: results from the continuous NHANES, 1999-2010. Am J Epidemiol. 2013;178:1106-13.

${ }^{1}$ Department of Gastroenterology, Changhai Hospital, Shanghai, China. ${ }^{2}$ Department of Gastroenterology, Gongli Hospital,

The Second Military Medical University, Shanghai, China. Correspondence: Z.-S.L. (email: zhaoshen-li@hotmail.com ) or L.-H.H. (email: lianghao-hu@hotmail.com)

\section{Response to Hao et al.}

Gregory A. Cote, MD MS'1, Dhiraj Yadav, MD $\mathrm{MPH}^{2}$ and Timothy B. Gardner, MD MS ${ }^{3}$

Am J Gastroenterol https://doi.org/10.1038/s41395018-0375-2

To the Editor: We thank Drs. Hao, Li and $\mathrm{Hu}$ [1] for their comments on our recent article published on the effect of recurrent acute pancreatitis in the absence of overt chronic pancreatitis (RAP) on quality of life [2]. We appreciate the opportunity to respond on behalf of our co-authors.

In respect to the first point regarding the heterogenous definition of RAP, we agree that there are different intervals between attacks used to define RAP. For the purposes of the North American Pancreatitis Study 2 (NAPS2), the source of data for this study, RAP was determined at the discretion of the treating physician; therefore, the interval between attacks is not known [3]. However, as all of the enrolling physicians in the NAPS consortium are expert pancreatologists and the definition of chronic pancreatitis was quite rigorous, 